\title{
Open charm production and spectroscopy at ATLAS and CMS
}

George W.S. Hou on behalf of the ATLAS and CMS Collaborations*

National Taiwan University

E-mail: wshoulphys.ntu.edu.tw

\begin{abstract}
The central and general purpose experiments at the LHC have contributed to open charm physics, complementing $\mathrm{LHCb}$ in $\mathrm{B}_{\mathrm{c}}$ property studies, and ATLAS has discovered a $\mathrm{B}_{\mathrm{c}}$ excitation that is consistent with $\mathrm{B}_{\mathrm{c}}(2 \mathrm{~S})$. ATLAS has studied charged $\mathrm{D}_{(\mathrm{s})}$ meson production, while CMS has studied $\mathrm{D}^{0}$ production. In particular, CMS measured the nuclear modification factor $R_{\mathrm{AA}}$ in $\mathrm{PbPb}$ collisions at $5.02 \mathrm{TeV}$, finding strong medium suppression in $\mathrm{PbPb}$ compared with pp collisions over a broad range of $p_{T}$, and consistent with the ALICE result scaled to similar energy. An important tool has been developed to identify charm jets, complementing b-tagging algorithms. CMS has developed a 2D c-tagger to discriminate c-jet from light jet and b-jet, respectively. After training on simulated data, the c-tagger has been validated with $\mathrm{W}+\mathrm{c}$ and $\mathrm{t} \overline{\mathrm{t}}$ events using 2015 data at $13 \mathrm{TeV}$, with extracted scale factor $S F_{\mathrm{c}}$ close to 1 . A similar c-tagger has been developed by ATLAS, but the 2D version for Run 2 is not yet publicly available.
\end{abstract}

VIII International Workshop On Charm Physics

5-9 September, 2016

Bologna, Italy

${ }^{*}$ Speaker. 


\section{What this talk is not}

To conform with the charge given me, let me up-front state what this talk is not about:

- This talk does not cover LHCb. ATLAS [1] and CMS [2] are central detectors, which lack K/ $\pi$ identification ability. Instead, the relevant strengths are: tracking (jets, hadron and vertex reconstruction) and muons. There is a dedicated LHCb talk [4], plus a general talk that also covers ALICE [5].

- Although a $\mathrm{B}^{+}$cross section (13 TeV) paper by CMS just appeared [3], which utilizes $\mathrm{J} / \psi \mathrm{K}^{+}$ final state, this talk is not about $\mathrm{B}$;

- Although e.g. CMS has once again rediscovered usual neutral vector mesons, from $\omega$ to $\Upsilon(\mathrm{nS})$ (and $\mathrm{Z}$ boson) at $13 \mathrm{TeV}$, this talk is not about onia;

But 2016 has been a very good year into LHC Run 2. As of Sept. 1st, LHC delivered close to $28 \mathrm{fb}^{-1}\left(41 \mathrm{fb}^{-1}\right.$ by end of $2016 \mathrm{pp}$ run), with ATLAS and CMS more than $90 \%$ efficient. In the following, we start from open charm spectroscopy, then turn to open charm production. Reflecting the interest even in the opening session of this CHARM conference, a good fraction of our time is spent discussing charm-jet tagging as an emerging new tool, before we close in a summary.

\section{Bc studies}

Though a little dated, the only contribution to open charm spectroscopy is the discovery, by ATLAS with both 7 and $8 \mathrm{TeV}$ data, of an excitation of the $\mathrm{B}_{\mathrm{C}}$ meson at $6842 \pm 4 \pm 5 \mathrm{MeV}$ that is consistent with the $\mathrm{B}_{\mathrm{c}}(2 S)$ state [6]. Detection is through reconstruction of $\mathrm{B}_{\mathrm{c}}^{ \pm} \rightarrow \mathrm{J} / \psi \pi^{+}$that resonates with a $\pi^{+} \pi^{-}$pair. Compared with $\psi(2 \mathrm{~S})$ and $\Upsilon(2 \mathrm{~S})$, a $\mathrm{B}_{\mathrm{C}}(2 \mathrm{~S})$ state that decays to $\mathrm{B}_{\mathrm{C}} \pi^{+} \pi^{-}$ has to exist, but this ATLAS state needs confirmation from LHCb and CMS.

Other than the excited $\mathrm{B}_{\mathrm{c}}$ state, both ATLAS and CMS have measured some $\mathrm{B}_{\mathrm{c}}$ properties. In a slightly dated paper, CMS measured [7]

$$
\left.\left.R_{\mathrm{c} / \mathrm{u}}=\frac{\sigma\left(\mathrm{B}_{\mathrm{C}}^{+}\right) \mathscr{B}\left(\mathrm{B}_{\mathrm{C}}^{+} \rightarrow \mathrm{J} / \psi \pi^{+}\right)}{\sigma\left(\mathrm{B}^{+}\right) \mathscr{B}\left(\mathrm{B}^{+} \rightarrow \mathrm{J} / \psi K^{+}\right)}=[0.48 \pm 0.05 \text { (stat. }) \pm 0.03 \text { (syst. }\right) \pm 0.05\left(\tau_{\mathrm{B}_{\mathrm{c}}}\right)\right] \%,
$$

complementary in kinematic region vs $\mathrm{LHCb}$, i.e. for higher $p_{T}>15 \mathrm{GeV}$ and more central $|y|<$ 1.6. CMS also measured

$$
\left.R_{\mathrm{B}_{\mathrm{c}}}=\frac{\mathscr{B}\left(\mathrm{B}_{\mathrm{c}}^{+} \rightarrow \mathrm{J} / \psi \pi^{+} \pi^{+} \pi^{-}\right)}{\mathscr{B}\left(\mathrm{B}_{\mathrm{C}}^{+} \rightarrow \mathrm{J} / \psi \pi^{+}\right)}=2.55 \pm 0.80 \text { (stat.) } \pm 0.33 \text { (syst. }\right)_{-0.01}^{+0.04}\left(\tau_{\mathrm{B}_{\mathrm{c}}}\right)
$$

confirming the result of $\mathrm{LHCb}$, which has better statistics.

More recently, ATLAS has studied $\mathrm{B}_{\mathrm{C}} \rightarrow \mathrm{J} / \psi \mathrm{D}_{\mathrm{S}}^{(*)}$ decays [8], including transverse polarization fraction for $\mathrm{B}_{\mathrm{c}} \rightarrow \mathrm{J} / \psi \mathrm{D}_{\mathrm{S}}^{*}$. and compared with $\mathrm{LHCb}$ (which has better errors) and theory. The results are basically consistent.

So one can see that the standard bearer is LHCb for open charm spectroscopy and properties. Note also that, for all the studies mentioned, one relies on $\mathrm{J} / \psi$ as part of final state reconstruction. We therefore happily recall that this year is the 40th anniversary for the Nobel prize to Burt Richter and Sam Ting, while $\mathrm{J} / \psi$ itself is 42 years and going, and would remain an important tool for years to come. 


\section{Open charm production}

Before considering open charm production, let us contrast with open beauty production. A bjet is typically tagged by a secondary vertex, and B-hadron production studies are often conducted with non-prompt $\mathrm{J} / \psi \rightarrow \mu^{+} \mu^{-}$as a tool, with the advantage of $\mathrm{c} \tau(\mathrm{B}) \sim 400$ to $500 \mu \mathrm{m}$. In comparison, c-jet tagging is more difficult (next section), and $\mathrm{D}$ hadron production has lower multiplicity, without the luxury of $\mathrm{J} / \psi$ assistance, and has shorter $\mathrm{c} \tau(\mathrm{D}) \sim 100$ to $300 \mu \mathrm{m}$. In the following, we report on $\mathrm{D}^{(*)+}$ and $\mathrm{D}_{\mathrm{S}}^{+}$production studies by ATLAS in pp collisions, and $\mathrm{D}^{0}$ production in $\mathrm{pp}$ and $\mathrm{PbPb}$ collisions by $\mathrm{CMS}$. Charge conjugate states are always implied.

\section{1 $\mathrm{D}^{(*)+}$ and $\mathrm{D}_{\mathrm{S}}^{+}$production in pp by ATLAS}

ATLAS has studied $\mathrm{D}^{(*)+}$ and $\mathrm{D}_{\mathrm{S}}^{+}$production in pp collisions at $7 \mathrm{TeV}$ with $280 \mathrm{nb}^{-1}$ data [9], with the reconstructions $\mathrm{D}^{*+} \rightarrow \mathrm{D}^{0}\left(\rightarrow \mathrm{K}^{+} \pi^{-}\right) \pi_{s}^{+}$, where $\pi_{s}^{+}$is the "soft" pion descendent from $\mathrm{D}^{*+} ; \mathrm{D}^{+} \rightarrow \mathrm{K}^{+} \pi^{-} \pi^{+} ; \mathrm{D}_{\mathrm{S}}^{+} \rightarrow \phi\left(\rightarrow \mathrm{K}^{+} \mathrm{K}^{-}\right) \pi^{+}$. These were for the kinematic range of $p_{T} \in$ (3.5, 100) $\mathrm{GeV}$ and $|\eta|<2.1$ for the reconstructed $D$ meson. Altogether, about 2900, 3700, $500 \mathrm{D}^{*+}, \mathrm{D}^{+}, \mathrm{D}_{\mathrm{S}}^{+}$mesons, respectively, were reconstructed.
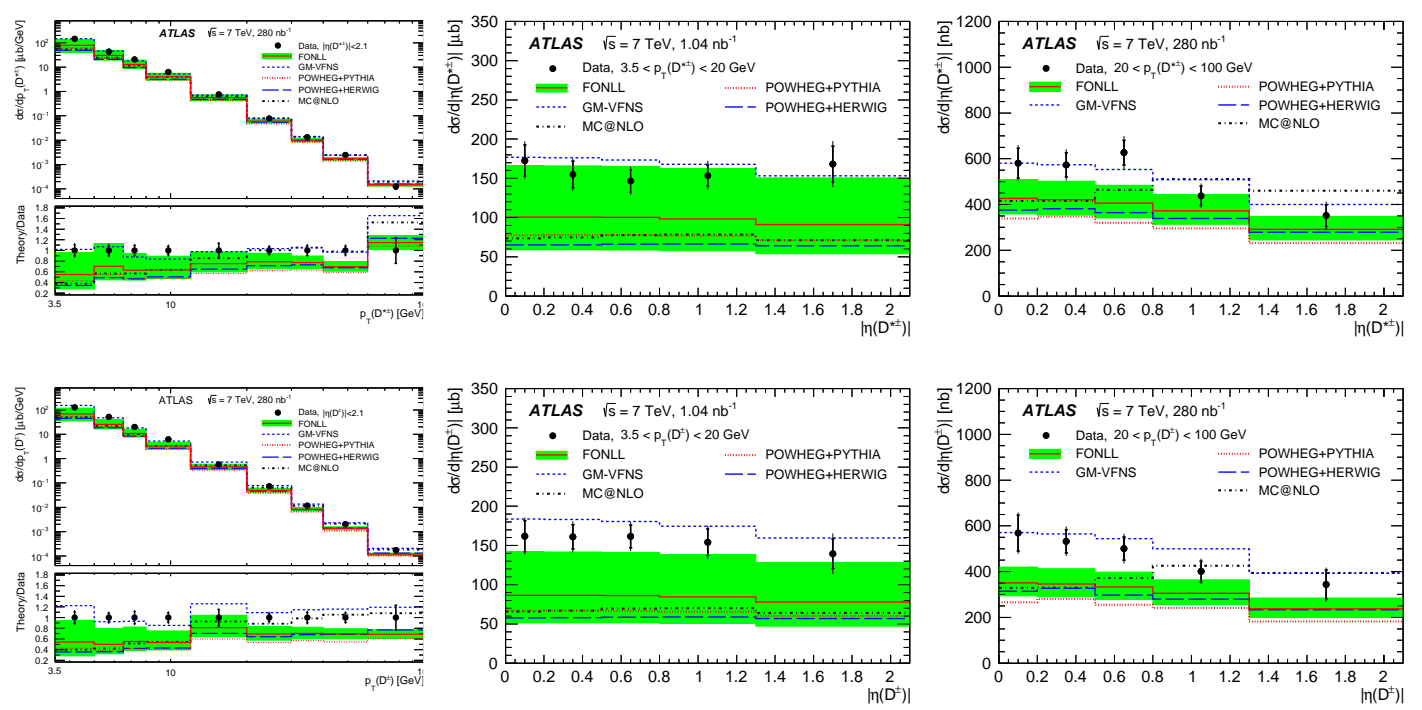

Figure 1: $d \sigma / d p_{T}$ and $d \sigma / d|\eta|$ for [9] the $p_{T}$ regions of $(3.5,20)$ and (20 100) $\mathrm{GeV}$, for $\mathrm{D}^{*+}$ (top) and $\mathrm{D}^{+}$ (bottom), compared with various NLO QCD calculations, with the FONLL range marked in green.

The differential $p_{T}$ and rapidity distributions, $d \sigma / d p_{T}$ and $d \sigma / d|\eta|$, are given in Fig. 1 for the two $p_{T}$ regions of $(3.5,20)$ and $(20100) \mathrm{GeV}$. Comparison is made with various NLO QCD calculations. While there is general agreement, taking the up to date fixed-order next-to-leadinglogarithm (FONLL) results $[9,10]$, data appear to be at upper limits of theory. Assuming FONLL to extrapolate to full kinematic space, ATLAS finds,

$$
\sigma_{\mathrm{C} \overline{\mathrm{C}}}^{\mathrm{tot}}=8.6 \pm 0.3(\mathrm{stat}) \pm 0.7(\mathrm{syst}) \pm 0.3(\mathrm{lum}) \pm 0.2(\mathrm{ff})_{-3.4}^{+3.8}(\mathrm{extr}) \mathrm{mb} \quad(\text { ATLAS })
$$


where "ff" marks the uncertainty due to fragmentation fraction, and "extr" for extrapolation procedure. This is consistent with the ALICE finding of [11]

$$
\sigma_{\mathrm{C} \overline{\mathrm{C}}}^{\text {tot }}=8.5 \pm 0.5 \text { (stat) }{ }_{-2.4}^{+1.0}(\text { syst }) \pm 0.3(\mathrm{lum}) \pm 0.2(\mathrm{ff})_{-0.4}^{+5.0} \text { (extr) mb (ALICE) }
$$

Note that the visible kinematic ranges for the two experiments are different, resulting in different extrapolation uncertainties.

\subsection{Prompt $\mathrm{D}^{0}$ production in pp and $\mathrm{PbPb}$ by $\mathrm{CMS}$}

CMS has made the first measurement of prompt $\mathrm{D}^{0}$ cross section in pp collisions at 5.02 $\mathrm{TeV}$ [12], and corresponding production in $\mathrm{PbPb}$ collisions at the same energy of $\sqrt{S_{N N}}=5.02 \mathrm{TeV}$. We illustrate in Fig. 2 the reconstruction of $\mathrm{D}^{0} \rightarrow \mathrm{K}^{+} \pi^{-}$events in pp and $\mathrm{PbPb}$ collisions, as well as the differential $d \sigma / d p_{T}$ distribution for the pp case, compared with theory calculations. One can see that, while there is agreement with theory, similar to $\mathrm{D}^{+}$production studied by ATLAS [9], data is at upper side of FONLL predictions.
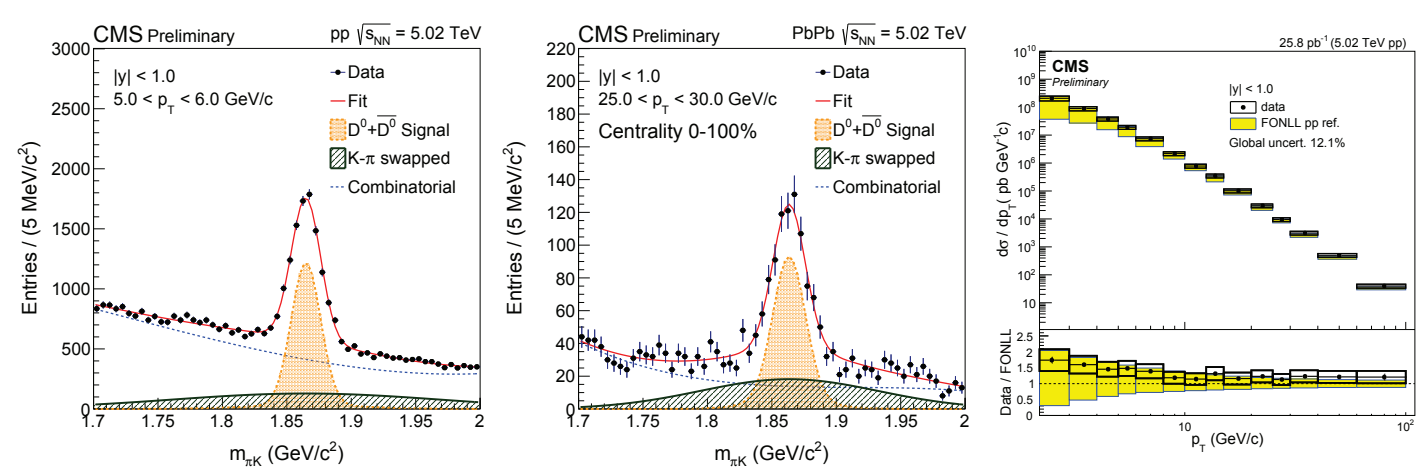

Figure 2: $\mathrm{D}^{0}$ candidate invariant mass distribution in $\mathrm{pp}$ (left) and $\mathrm{PbPb}$ (center) collisions at $5.02 \mathrm{TeV}$ and the differential $d \sigma / d p_{T}$ distribution (right) for the former [12], compared with FONLL predictions.

The real aim of the CMS study, however, is to extract the nuclear modification factor $R_{\mathrm{AA}}$ for $\mathrm{D}^{0}$ production, by comparing $\mathrm{PbPb}$ to pp collisions. As discussed in talk by Geurts [13], one probes energy loss as the $\mathrm{D}^{0}$ travels through, and strongly interacts with, the nuclear medium. To quantify medium effects, CMS defines

$$
R_{\mathrm{AA}}\left(p_{T}\right)=\frac{1}{T_{\mathrm{AA}}} \frac{d N_{\mathrm{PbPb}}^{\mathrm{D}^{0}}}{d p_{T}} / \frac{d N_{\mathrm{pp}}^{\mathrm{D}^{0}}}{d p_{T}},
$$

for $|y|<1.0$, where $T_{\mathrm{AA}}$ is the nuclear overlap function, which is related to $N_{\text {coll }}$ (number of incoherent nucleon-nucleon collisions) defined through the ratio of double-differential cross sections [13]. In an earlier study by CMS at $\sqrt{S_{\mathrm{NN}}}=2.76 \mathrm{TeV}$ [14], the nuclear modification factor of prompt $\mathrm{D}^{0}$ cross section in $\mathrm{PbPb}$ collisions, called $R_{\mathrm{AA}}^{*}$ in that study (because the pp reference is extrapolated through FONLL), was found quite suppressed for $p_{T} \sim 5-10 \mathrm{GeV}$. As can be seen from Fig. 3, the suppression appears more prominent for the centrality class of $0-10 \%$, compared with the $0-100 \%$. The result is consistent with the ALICE $7 \mathrm{TeV}$ result [11], rescaled to $2.76 \mathrm{TeV}$ using FONLL.

To further study this effect with less theory dependence, CMS took $25.8 \mathrm{pb}^{-1}$ and $404 \mu \mathrm{b}^{-1}$ data for $\mathrm{pp}$ and $\mathrm{PbPb}$ collisions, respectively, both at $5.02 \mathrm{TeV}$ [12], and made multiple checks and 

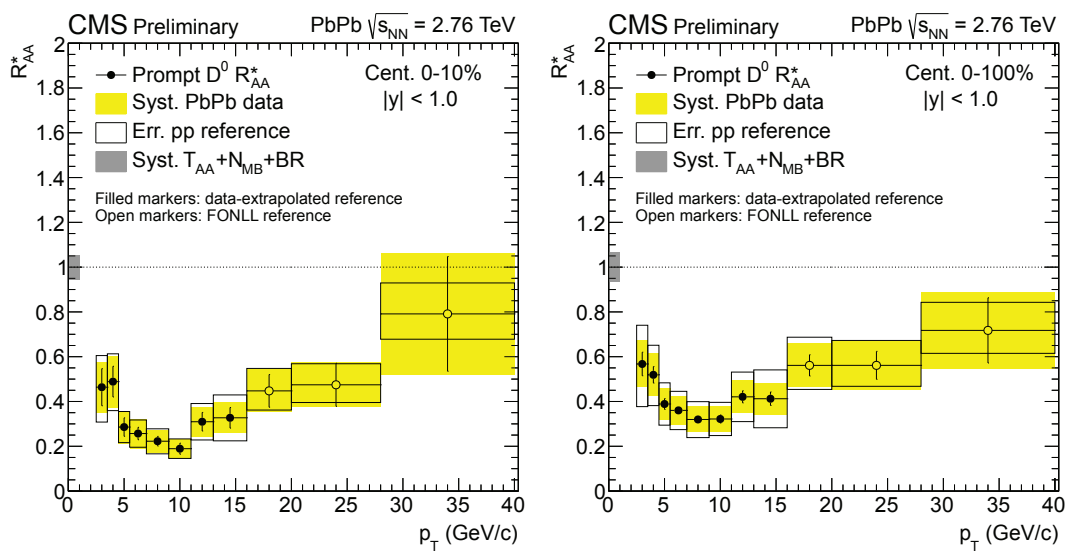

Figure 3: Nuclear modification factor $R_{\mathrm{AA}}^{*}$ (pp reference scaled by FONLL) vs $p_{T}$ for prompt $\mathrm{D}^{0}$ production in $\mathrm{PbPb}$ data at $2.76 \mathrm{TeV}$ [14], for centrality classes $0-10 \%$ (left) and 0-100\% (right).

comparisons, including the measurement of differential prompt $\mathrm{D}^{0}$ production $p_{T}$ distribution in pp collisions given in Fig. 2(right). We show in Fig. 4 the nuclear modification factor $R_{\mathrm{AA}}$ [12] for centrality classes $0-10 \%$ (left) and 0-100\% (right), which are rather busy plots. Let us not comment on the details of comparison with theory and other concerns, which populate the plot, but offer some generic remarks.

First, the suppression of $R_{\mathrm{AA}}$, or medium effect, is generic for $p_{T}$ above a few $\mathrm{GeV}$ to more than $10 \mathrm{GeV}$. Even for the centrality range $0-100 \%$, there is a factor of $4-5$ suppression at $p_{T} \sim$ 6-7 GeV. Second, this suppression weakens for higher $p_{T}$, decreasing to only a factor of 1.5 for $p_{T}$ in range of $60-100 \mathrm{GeV}$ for the centrality range $0-100 \%$. The large measured $p_{T}$ range gives some challenge to theory, where none could fully capture the observed features. Third, within uncertainties, the $\mathrm{D}^{0}$ nuclear modification factor is consistent with $R_{\mathrm{AA}}$ of inclusive charged hadron
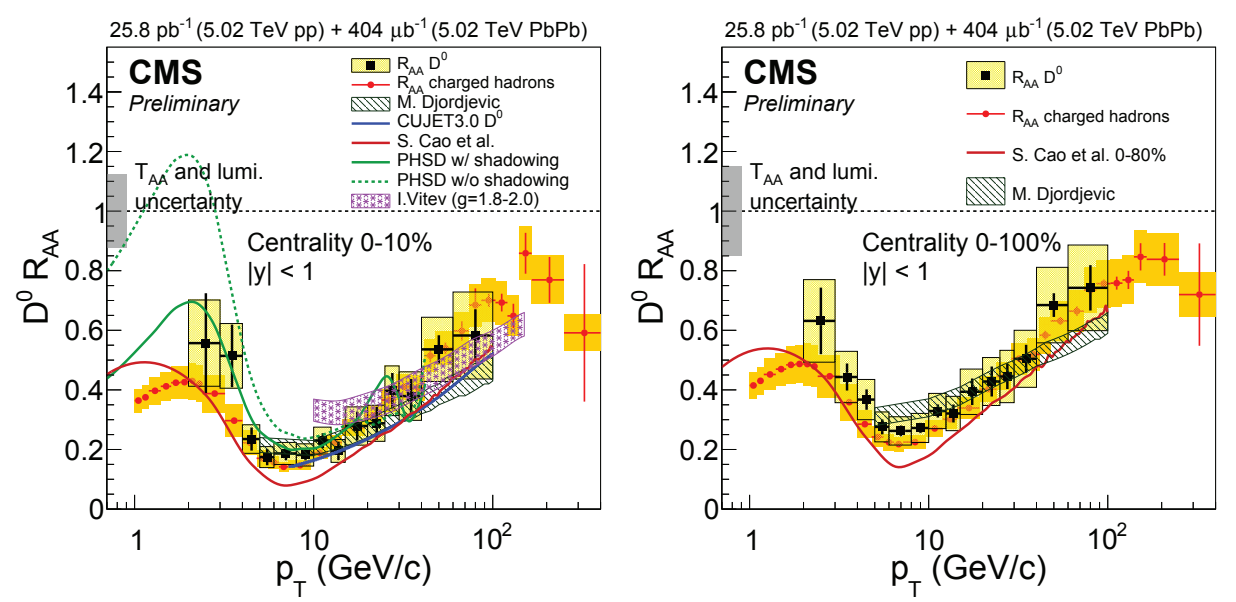

Figure 4: Nuclear modification factor $R_{\mathrm{AA}}$ vs $p_{T}$ for $\mathrm{D}^{0}$ production in $\mathrm{PbPb}$ at $5.02 \mathrm{TeV}$ [12], for centrality classes $0-10 \%$ (left) and 0-100\% (right), compared with various theories (see Ref. [12] for references). The orange boxes with data marked in red give $R_{\mathrm{AA}}$ for inclusive charged hadron production. 
production [15], shown as the orange boxes in Fig. 4. Finally, within errors, the $5.02 \mathrm{TeV}$ study is compatible with the results of the $2.76 \mathrm{TeV}$ study [14] given in Fig. 3, where the pp reference result was scaled through FONLL. Similarly, the 5.02 TeV result of CMS is compatible with the $2.76 \mathrm{TeV}$ result of ALICE (scaled with FONLL by CMS [14]) with smaller $|y|$ range.

\section{Charm-jet tagging: tool development}

If $\mathrm{D}$ meson production is a purpose and a means in itself, charm-jet tagging is a new frontier. As Lenz has raised [16] in his theory overview several times, and echoed by good interest in the audience, the $\mathrm{H} \rightarrow \mathrm{c} \overline{\mathrm{c}}$ decay has not yet been probed. Imagine what a c-tagger could do besides probing $\mathrm{H} \rightarrow \mathrm{c} \overline{\mathrm{c}}$ : one could study $\tilde{\mathrm{t}} \rightarrow \tilde{\chi}^{0}+\mathrm{c}$ in SUSY, or FCNC $\mathrm{t} \rightarrow \mathrm{c}+\mathrm{Z} / \gamma$ and FCNH $\mathrm{t} \rightarrow \mathrm{cH}$ processes. The latter is a new frontier in itself, as nothing forbids it from first principles, despite popular ad hoc assumptions [17] such as discrete symmetries in two Higgs doublet models.

But c-jet tagging is considerably more difficult than b-tagging, as alluded to at the beginning of the previous section. A c-jet presents characteristics which are in between a b-jet and light jet (originating from light partons: $\mathrm{u}, \mathrm{d}, \mathrm{s}$ quark or gluon), such as having tracks and vertices less displaced than a b-jet, but more than a light jet. The b-tagging algorithms will therefore select part of the c-jets. While b-tagging is quite well developed and exploited, a dedicated c-tagger needs to exploit detailed c-jet properties versus b- and light jets.

\subsection{Charm-tagged jet production in $\mathrm{pPb}$ and $\mathrm{pp}$ collisions by $\mathrm{CMS}$}

It is interesting that a study of charm-jets was performed on actual data involving heavy ions. CMS has made a first measurement of charm-jets in $\mathrm{pPb}$ collisions at $5.02 \mathrm{TeV}$ [18], and compared it with pp collisions at $2.76 \mathrm{TeV}$. Charm-jets are identified by requiring a secondary vertex comprised of three or more charged tracks that are significantly displaced from the primary vertex. A variant of the secondary vertex mass is used to extract the relative contributions of jet flavors. It was found that jet energy modification in $\mathrm{pPb}$ collisions is consistent with pp collisions, even though comparison was made at different collision energies. Furthermore, the charm-jet fraction is
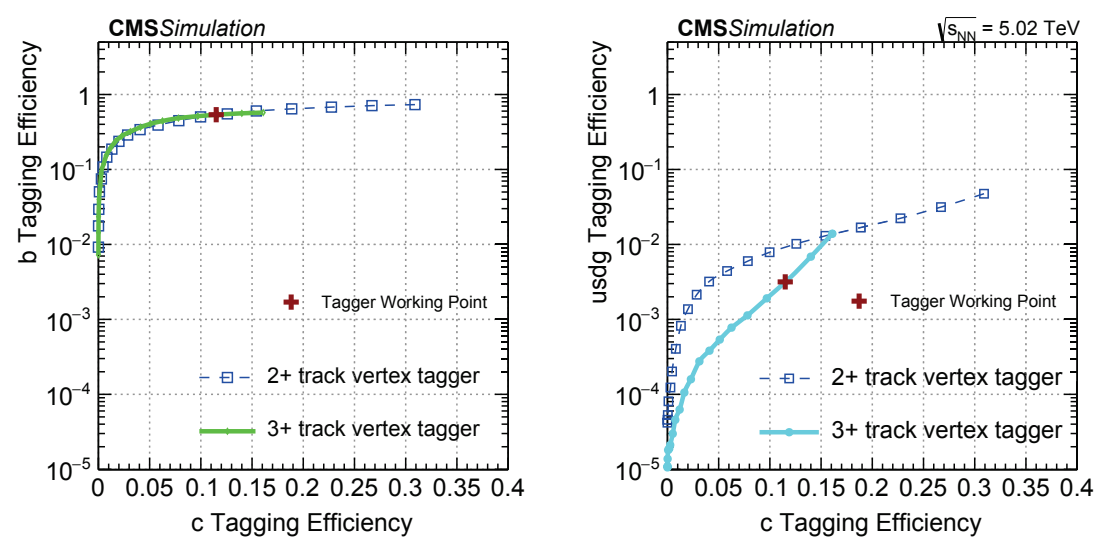

Figure 5: Simple secondary vertex (SSV) tagger for both b (left) and light (right) jets, as a function of c-jet tagging efficiency [18]. 
consistent with PYTHIA within uncertainties for both $\mathrm{pPb}$ and $\mathrm{pp}$ collisions. The results are being updated towards a paper.

Our point of discussion, however, is to compare the charm-jet tagging with a new c-tagger discussed in Sec. 4.3. The study of Ref. [18] uses a variant of the b-tagger, namely [19] simple secondary vertex (SSV), high purity (HP), and is not a dedicated c-jet tagger. To elaborate, we plot [18] SSV for b-jet and light quark jet vs c-jet tagging efficiency in Fig. 5. From the left plot we see the charm-to-bottom discrimination is basically unchanged. However, light jet mistag is reduced by a factor of 3 at the marked point on the blue curve of the right plot.

\subsection{Charm-jet tagging at ATLAS}

Before we present developments at CMS on a dedicated c-tagger, let us comment on the status at ATLAS. ATLAS has been designing [20] a charm-tagging algorithm, called JetFitterCharm, based on $8 \mathrm{TeV}$ studies for both simulation and data. For $13 \mathrm{TeV}$ at LHC Run 2, the IBL (Insertable B-Layer) would enhance performance, and ATLAS is developing a 2D optimization towards a dedicated c-tagger. Basically, it uses two BDTs where one is trained for c-jet against light jet, and one for c-jet against b-jet. While there is no public update yet, most ingredients can be found on ATLAS Flavour Tagging public pages. For example, b-jet (and c- and light jet) performance plots at $13 \mathrm{TeV}$ can be seen in Ref. [21], while both c-jet and light jet calibrations at $8 \mathrm{TeV}$ are described in Ref. [22].

One important issue is the validation of charm-jet mistag. ATLAS has measured [23], using the b-tagger, charm mistag with $\mathrm{W}+\mathrm{c}$ production at $7 \mathrm{TeV}$. Based on this work, a poster [24] was presented at ICHEP2016, where ATLAS promises that a similar measurent with $13 \mathrm{TeV}$ data is "expected soon".

\subsection{Charm-jet tagging developments at CMS}

In the following, we present the CMS $13 \mathrm{TeV}$ c-tagging tool development based on 2015 data. A poster and a parallel session talk were also presented [25].

For Run2 [26], CMS provides two main b-taggers: CSVv2 and cMVAv2. The first one is an optimised version with respect to Run1, and combines information about tracks and secondary vertices (SV). The second one adds another layer and combines with a multivariate analysis approach the output of CSVv2 with other taggers like Jet Probability (based only on track information) or the Soft Lepton Taggers (based on the presence of a soft lepton (SL) within the jet). For the c-tagger developed [27] for Run 2, which uses 2015 data for calibrating the algorithm, one simplifies and makes a straightforward combination of tracks, SV and SL info.

The issue at hand is to separate c-jet from b-jet as well as light jet backgrounds. The solution is to have two BDTs: c- vs light (CvsL) jets and c- vs b- $(\mathrm{CvsB})$ jets [27]. The c-tagger is trained on simulated QCD multijets, but the performance is also validated on simulated $t \bar{t}$ samples. The corresponding performance [27] are given in Fig. 6. The spikes in discriminator shapes on the left figures appear for jets in which no track passes the specific selection criteria [27]. For the final performance displayed in Fig. 6(right), results from current cMVAv2 and CSVv2 b-tagger algorithms [26] are also shown for comparison. Here, the blue curves correspond to the scales in blue on the left for light-jet efficiency, while the red curves correspond to $b$ jet efficiencies and refer 

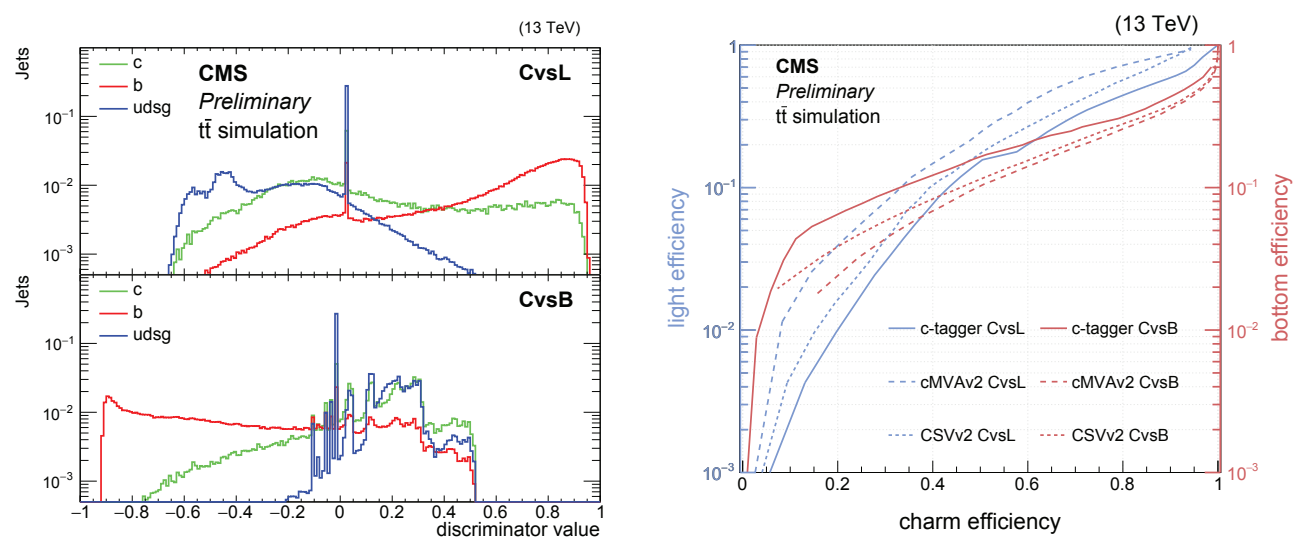

Figure 6: Left [27]: CvsL (top) and CvsB (bottom) discriminators, normalized for each flavour. Right [27]: final performance of CvsL (blue solid line and axis) and CvsB (red solid line and axis) validated on $\bar{t}$, and compared with using various b-tagging algorithms (dash and dotted lines).
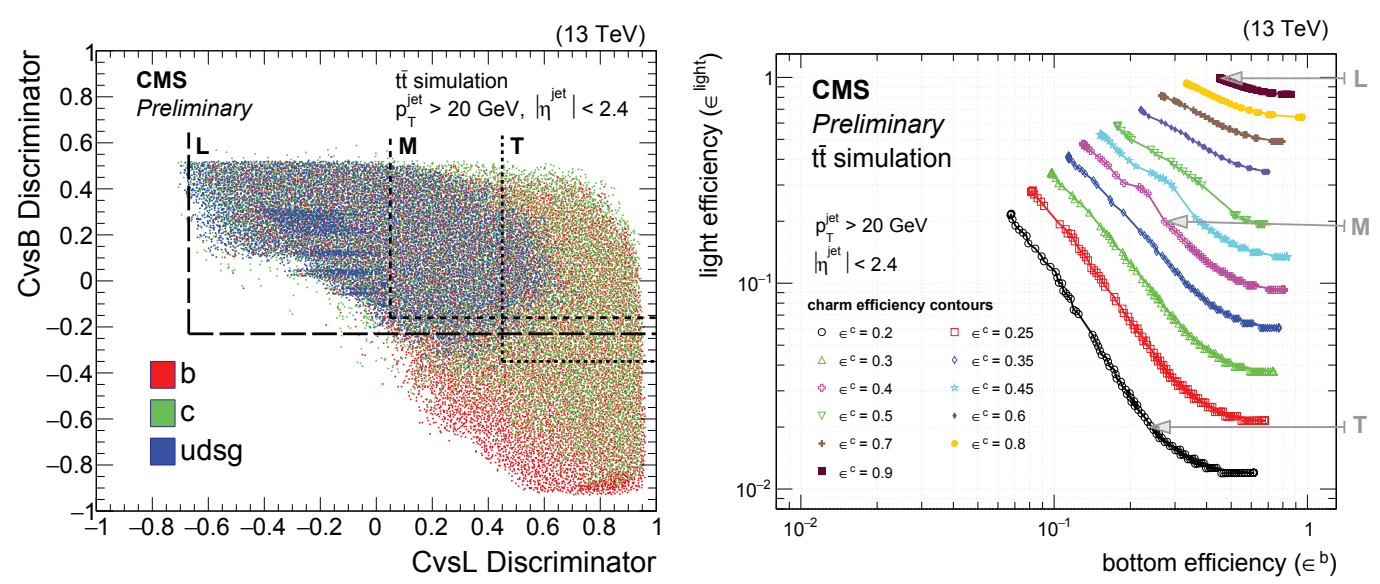

Figure 7: Left [27]: 2D scatter for b (red), c (green), and light (blue) jets, in the plane of CvsL and CvsB. Right [27]: contours in plane of bottom and light mistag efficiencies for different values of constant charm efficiency. The markers $\mathrm{L}, \mathrm{M}$ and $\mathrm{T}$ are explained in text.

to the scale on the right. Just like in Fig. 5, closer to the lower right means better performance. One sees that, compared with current b-tagger tools, CvsL discriminates better between light and charm jets, while CvsB performs worse. The latter is due to the fact that this first implementation of the tagger focused on rejecting light flavor jets. An improved version of the tagger, with a stronger separation between c-jets and b-jets, is being developed.

We show in Fig. 7 (left) the distribution of jets of different flavours in the plane formed by the two discriminators [27]. The two classifiers output a value close to $1(-1)$ for signal-like (background-like) jets. Thus $\mathrm{c}$ jets will be located towards the upper right corner, while $\mathrm{b}$ and light jets populate mostly the bottom right and the top left corners, respectively. Contours of constant charm efficiency are shown in Fig. 7(right) in the plane of light and b-jet mistag efficiencies. To separate c-jets from the background, a rectangular cut is placed to isolate the upper right corner in Fig. 7(left). Three such cuts, L(oose), M(edium) and T(ight) are marked, which correspond to the 
working points (WP) marked in Fig. 7(right). In particular, for c-tagger T (ctagT), one has CvsL $>0.45, \mathrm{CvsB}>-0.35$, and $\varepsilon^{\mathrm{c}}, \varepsilon^{\mathrm{b}}, \varepsilon^{\text {light }}=0.2,0.24,0.02$, respectively.

We use ctagT to illustrate the validation on data [27] of the charm tagger algorithm. Two methods are used. The first uses the very pure sample of c-jets from $\mathrm{g}+\mathrm{s}(\mathrm{d}) \rightarrow \mathrm{W}^{-}+\mathrm{c}$. Since the signal W and c charges are correlated, while QCD background of ce pair production in association with a $\mathrm{W}$ is not, subtracting same-sign from opposite-sign events effectively eliminates the latter. A second validation utilizes semileptonic decay of a t't pair, where roughly $25 \%$ of jets are c-jets. Utilizing weak decay properties that boost u-type quarks, one can infer the efficiency of the charmtagging algorithm. From these data vs simulation validations, one measures the scale factors, or ratio of efficiencies,

$$
S F_{\mathrm{c}}=\varepsilon_{\mathrm{c}}^{\mathrm{data}} / \varepsilon_{\mathrm{c}}^{\mathrm{MC}}
$$

as depicted in Fig. 8 for the working point ctagT. Validation uses $13 \mathrm{TeV}$ data of 2015 . The $\mathrm{W}+\mathrm{c}$ validation provides four momentum bins, while the $\mathrm{t} \overline{\mathrm{t}}$ validation is not binned in transverse momentum of the jet due to statistical limitations. The extracted scaling factor is close to 1 [27].
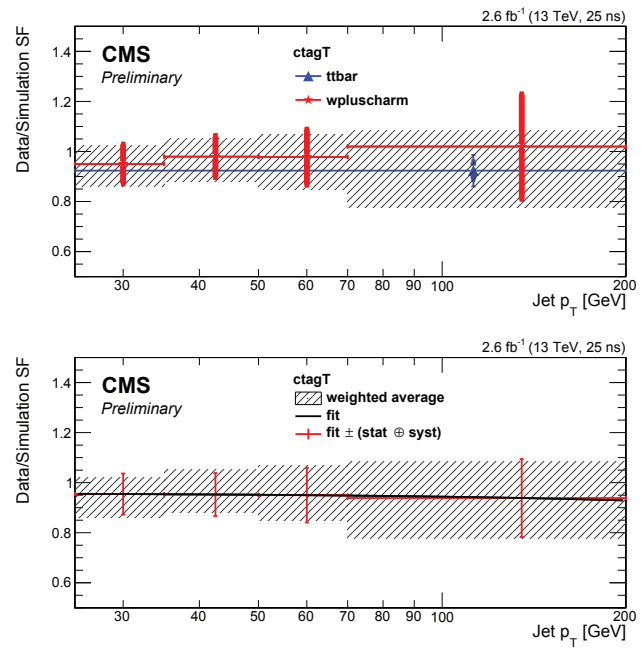

Figure 8: Data-to-simulation scale factor SF of the c-tagging efficiency for working point c-tagger Tight [27]. Upper panel gives measured values by two methods, with (thick error bar) statistical error and (narrow error bar) combined statistical and systematic uncertainties. The hatched area is the combined SF value with overall uncertainty, displayed again in lower panel with solid line as the linear fit.

\section{Summary}

Although ATLAS and CMS are central, general purpose detectors, they have contributed to open charm spectroscopy, such as finding an excited $\mathrm{B}_{\mathrm{C}}$ state, and explored $\mathrm{B}_{\mathrm{C}}$ meson properties. Both experiments have studied prompt $\mathrm{D}$ meson production in pp collisions, while CMS has measured the Nuclear Modification Factor $R_{\mathrm{AA}}$. For prompt $\mathrm{D}^{0}$ production in $\mathrm{PbPb}$ vs pp collisions, strong suppression is observed for $p_{T}$ ranging from a few $\mathrm{GeV}$ to over $10 \mathrm{GeV}$. This mediuminduced suppression for prompt $\mathrm{D}^{0}$ is consistent with what is observed for inclusive charged parti- 
cle production. The high $p_{T}$ capability allows one to probe up to $100 \mathrm{GeV}$ for $\mathrm{D}^{0}$, and hundreds of $\mathrm{GeV}$ for charged hadrons.

Following the great impact of b-tagging algorithms, ATLAS and CMS are developing dedicated charm-jet taggers for LHC Run 2. For example, for the first time at CMS, a 2D c-tagger is validated with $13 \mathrm{TeV}$ data of 2015 , with room for further improvement. ATLAS has similar developments, but the 2D c-tagger for Run 2 is not yet public. Let's hope we would soon hear from actual applications in future analyses.

\section{References}

[1] ATLAS Collaboration, JINST 3 S08003 (2008).

[2] CMS Collaboration, JINST 3 S08004 (2008).

[3] V. Khachatryan et al. [CMS Collaboration], arXiv:1609.00873 [hep-ex].

[4] P. Spradlin, this proceedings.

[5] A. Mischke, this proceedings.

[6] ATLAS Collaboration, Phys. Rev. Lett. 113, 212004 (2014) [arXiv:1407.1032 [hep-ex]].

[7] V. Khachatryan et al. [CMS Collaboration], JHEP 1501, 063 (2015) [arXiv:1410.5729 [hep-ex]].

[8] ATLAS Collaboration, Eur. Phys. J. C 76, 4 (2016) [arXiv:1507.07099 [hep-ex]].

[9] ATLAS Collaboration, Nucl. Phys. B 907, 717 (2016) [arXiv:1512.02913 [hep-ex]].

[10] M. Cacciari et al., JHEP 1210, 137 (2012); for FONLL webpage update, see Ref. [9].

[11] B. Abelev et al. [ALICE Collaboration], JHEP 1201, 128 (2012) [arXiv:1111.1553 [hep-ex]].

[12] CMS PAS HIN-16-001 [CMS Collaboration].

[13] F. Geurts, this proceedings.

[14] CMS PAS HIN-15-005 [CMS Collaboration].

[15] V. Khachatryan et al. [CMS Collaboration], arXiv:1611.01664 [nucl-ex].

[16] A. Lenz, this proceedings.

[17] S. L. Glashow and S. Weinberg, Phys. Rev. D 15, 1958 (1977).

[18] CMS PAS HIN-15-012 [CMS Collaboration].

[19] S. Chatrchyan et al. [CMS Collaboration], JINST 8, P04013 (2013) [arXiv:1211.4462 [hep-ex]].

[20] ATL-PHYS-PUB-2015-001 [ATLAS Collaboration].

[21] See http://atlas.web.cern.ch/Atlas/GROUPS/PHYSICS/PLOTS/FTAG-2016-001/

[22] ATLAS-CONF-2014-046 [ATLAS Collaboration].

[23] ATLAS-CONF-2013-109 [ATLAS Collaboration].

[24] Poster presented by A. Lapertosa at ICHEP2016, August 2016, Chicago, USA.

[25] Poster and parallel session talk by S. Moortgat, this proceedings.

[26] CMS PAS BTV-15-001 [CMS Collaboration].

[27] CMS PAS BTV-16-001 [CMS Collaboration]. 